\title{
Ablation vs Medication as Initial Therapy for Paroxysmal Atrial Fibrillation: An Updated Meta-Analysis of Randomized Controlled Trials
}

Jakrin Kewcharoen ${ }^{1}$, Narut Prasitlumku², Ronpichai Chokesuwattanaskul ${ }^{3}$, Ruiyang $\mathrm{Yi}^{4}$, Krit Jongnarangsin ${ }^{5}$, Thomas Bunch ${ }^{6}$, Ravi Ranjan ${ }^{7}$, and Leenhapong Navaravong ${ }^{6}$

${ }^{1}$ University of Hawaii Internal Medicine Residency Program

${ }^{2}$ University of California Riverside

${ }^{3}$ King Chulalongkorn Memorial Hospital

${ }^{4}$ University of Hawai'i at Manoa John A Burns School of Medicine

${ }^{5}$ University of Michigan

${ }^{6}$ University of Utah School of Medicine

${ }^{7}$ The University of Utah School of Medicine

March 30, 2021

\begin{abstract}
Background: Recent randomized controlled trials (RCT) suggest that ablation is superior to antiarrhythmic drugs (AAD) as an initial therapy for paroxysmal atrial fibrillation ( $\mathrm{pAF}$ ) to prevent arrhythmia recurrences. We performed an updated metaanalysis of RCTs, to include recent data from cryoballoon-based ablation, and to compare arrhythmia-free survival and adverse events between ablation and AAMs. Methods: We searched MEDLINE and EMBASE from inception to December 2020. We included RCT comparing patients with pAF undergoing ablation or receiving AADs as an initial therapy. We combined data using the random-effects model to calculate hazards ratio (HR) for arrhythmia-free survival and odds ratio (OR) for adverse events. Results: Five studies from 2005-2020 involving 985 patients were included (495 patients and 490 patients underwent ablation and medication as initial therapy, respectively). Patients who underwent ablation had higher freedom from atrial tachyarrhythmias (AT) during the 12-24 months follow-up period (pooled HR=0.48, 95\% CI:0.40-0.59, p <0.001) (Figure 2). In a subgroup analysis of ablation method used, both cryoablation group (pooled HR=0.49, 95\% CI:0.38-0.64, p <0.001) (Figure $2 \mathrm{~A}$ ) and radiofrequency ablation group (pooled $\mathrm{HR}=0.47,95 \% \mathrm{CI}: 0.35-0.64, \mathrm{p}<0.001$ ) (Figure $2 \mathrm{~B}$ ) showed reduction in $\mathrm{AT}$ recurrence compared to AAD group. There were no differences in adverse events including cerebrovascular accident, pericardial effusion or tamponade, pulmonary vein stenosis, acute coronary syndrome, deep vein thrombosis and pulmonary embolism, and bradycardia requiring a pacemaker. Conclusion: Catheter ablation (both cryoablation and radiofrequency ablation) is superior to AAD as an initial therapy for pAF in efficacy for reducing AT recurrences without a compromise in adverse events.
\end{abstract}

\section{Ablation vs Medication as Initial Therapy for Paroxysmal Atrial Fibrillation: An Updated Meta-Analysis of Randomized Controlled Trials}

Jakrin Kewcharoen $\mathrm{MD}^{1}$, Narut Prasitlumkum $\mathrm{MD}^{2}$, Ronpichai Chokesuwattanaskul $\mathrm{MD}^{3}$, Ruiyang $\mathrm{Yi}^{4}$, Krit Jongnarangsin $\mathrm{MD}^{5}$, $\mathrm{T}$ Jared Bunch $\mathrm{MD}^{6}$, Ravi Ranjan, $\mathrm{MD} \mathrm{PhD}^{6}$, Leenhapong Navaravong $\mathrm{MD}^{6}$

1) University of Hawaii Internal Medicine Residency Program, Honolulu, HI, USA

2) Division of Cardiology, University of California Riverside School of Medicine, Riverside, California, USA 
3) Division of Cardiology, Department of Medicine, Faculty of Medicine, Chulalongkorn University and King Chulalongkorn Memorial Hospital, Thai Red Cross Society, Bangkok, Thailand

4) Department of Internal Medicine, John A. Burns School of Medicine, Honolulu, HI, USA

5) Division of Cardiovascular Medicine, Department of Internal Medicine, University of Michigan Medical School, Ann Arbor, MI, USA

6) Division of Cardiovascular Medicine, Department of Internal Medicine, University of Utah School of Medicine, Salt Lake City, UT, USA

Running head: Ablation as initial therapy for paroxysmal atrial fibrillation

Keywords: atrial fibrillation ablation, first line therapy, paroxysmal atrial fibrillation

Acknowledgement: None

Financial support: None

Conflict of Interest : All authors declare no conflict of interest

\section{Address for correspondence:}

Leenhapong Navaravong, MD

Division of Cardiovascular Medicine

University of Utah School of Medicine

30 N 1900 E, 4A-100

Salt Lake City, UT, 84132

Email: l.navaravong@hsc.utah.edu

Word count: 2365

References: 22

Background: Recent randomized controlled trials (RCT) suggest that ablation is superior to antiarrhythmic drugs (AAD) as an initial therapy for paroxysmal atrial fibrillation (pAF) to prevent arrhythmia recurrences. We performed an updated meta-analysis of RCTs, to include recent data from cryoballoon-based ablation, and to compare arrhythmia-free survival and adverse events between ablation and AAMs.

Methods: We searched MEDLINE and EMBASE from inception to December 2020. We included RCT comparing patients with pAF undergoing ablation or receiving AADs as an initial therapy. We combined data using the random-effects model to calculate hazards ratio (HR) for arrhythmia-free survival and odds ratio $(\mathrm{OR})$ for adverse events.

Results: Five studies from 2005-2020 involving 985 patients were included (495 patients and 490 patients underwent ablation and medication as initial therapy, respectively). Patients who underwent ablation had higher freedom from atrial tachyarrhythmias (AT) during the 12-24 months follow-up period (pooled $\mathrm{HR}=0.48,95 \% \mathrm{CI}: 0.40-0.59, \mathrm{p}<0.001$ ) (Figure 2). In a subgroup analysis of ablation method used, both cryoablation group (pooled HR=0.49, 95\% CI:0.38-0.64, $\mathrm{p}<0.001$ ) (Figure 2A) and radiofrequency ablation group (pooled $\mathrm{HR}=0.47,95 \% \mathrm{CI}: 0.35-0.64, \mathrm{p}<0.001$ ) (Figure $2 \mathrm{~B}$ ) showed reduction in AT recurrence compared to AAD group. There were no differences in adverse events including cerebrovascular accident, pericardial effusion or tamponade, pulmonary vein stenosis, acute coronary syndrome, deep vein thrombosis and pulmonary embolism, and bradycardia requiring a pacemaker.

Conclusion: Catheter ablation (both cryoablation and radiofrequency ablation) is superior to AAD as an initial therapy for pAF in efficacy for reducing AT recurrences without a compromise in adverse events. 
Keywords: atrial fibrillation ablation; first line therapy; paroxysmal atrial fibrillation

\section{Introduction}

Atrial fibrillation (AF) is the most common sustained cardiac arrhythmia affecting approximately 1-2\% of the worldwide population [1]. Without appropriate treatment, AF can significantly impact quality of life with risks of recurrences and/or arrhythmia progression reported to be as high as $90 \%[2,3]$. Catheter ablation has shown to be an effective treatment with superior efficacy compared to antiarrhythmic drugs (AAD) alone for symptomatic AF $[4,5]$. The principle aim of AF ablation is to achieve durable circumferential pulmonary vein isolation (PVI), which electrically separates the pulmonary vein (PV) from the left atrium (LA) at the level of PV ostia/antrum. Although AF ablation is considered relatively safe, the procedure is invasive and carries risks of devastating complications such as esophageal-related injuries, pericardial effusion with tamponade, pulmonary vein stenosis, and cerebrovascular accident [6, 7]. Because of this, ablation is typically utilized in patients who failed initial AAD therapy, and most evidence supporting the use and the superiority of ablation was derived from populations that had already received an AAD as the first-line rhythm-based treatment $[8,9]$.

Similarly, previously published randomized controlled trials (RCTs) suggest that pulmonary vein isolation is superior to AAD even as initial therapy for paroxysmal atrial fibrillation (pAF). These include the Radiofrequency Ablation vs Antiarrhythmic Drugs as First-Line Treatment of Paroxysmal Atrial Fibrillation (RAAFT-2) trial, the Medical Antiarrhythmic Treatment or Radiofrequency Ablation in Paroxysmal Atrial Fibrillation (MANTRA-PAF) trial, and the Radiofrequency Ablation vs Antiarrhythmic Drugs as First-line Treatment of Symptomatic Atrial Fibrillation (RAAFT-1) trial [10-12]. A meta-analysis of the 3 RCTs by Hakalathi et al. confirmed this finding but reported that ablation was associated with more serious adverse events [13]. However, all 3 RCTs were done using only radiofrequency ablation without any trial performing cryoablation. Moreover, the authors did not perform time-to-event analysis for the main outcome of arrhythmic recurrence. In this updated meta-analysis, we included 2 recently published RCTs that use cryoballoon ablation, the Early Aggressive Invasive Intervention for Atrial Fibrillation (EARLY-AF) trial and the Cryoballoon Catheter Ablation in an Antiarrhythmic Drug Naive Paroxysmal Atrial Fibrillation (STOP AF First) trial and perform a sensitivity-analysis to compare arrhythmia-free survival and to evaluate adverse events between the two strategies $[14,15]$.

\section{Methods}

\subsection{Search strategy}

Two investigators (RY and NP) independently searched for published RCTs indexed in PubMed and EMBASE from inception to December 2020 using the search terms including: "atrial fibrillation", "ablation", and "initial" as described in supplementary file 1. Only articles in English were included. An additional manual search for potential additional pertinent studies was performed using the references from retrieved articles. Any conflict or discrepancy was resolved by a third author (LN)

\subsection{Inclusion criteria}

The inclusion criteria were as follows

(1) Randomized controlled trial conducted in patients with pAF comparing ablation and AAM as an initial therapy

(2) Studies must report recurrence rates of atrial tachyarrhythmias (AT) including AF, atrial flutter and atrial tachycardia, and adverse events following the index ablation in the ablation group and in the AAD group after randomization. Hazard ratio (HR), odds ratio (OR) or sufficient raw data to calculate effect size must be provided [16].

\subsection{Quality of included studies}

Cochrane Collaboration tool for assessing risk of bias was used to evaluate the quality of each randomized 
controlled trial by assigning a score (high, low, or unclear) for each individual element from five domains (selection, performance, attrition, reporting, and other) [17].

\subsection{Data extraction}

A standardized data collection form was used to obtain the following data from each study including name of the first author, year of publication, country of the study, study population, main inclusion and exclusion criteria, demographic data of participants, ablation procedure details, AAD therapy, endpoint for recurrence, recurrent rates, and reported adverse event.

To ensure accuracy, this data extraction process was independently performed by all investigators. Any data discrepancy was also resolved by referring back to the original articles.

\subsection{Statistical analysis}

We performed meta-analysis of included studies using a random-effects model and the generic inverse-variance method of Der Simonian and Laird to calculate pooled HR [18]. We extracted from these studies the freedom from AT rates and complications rates. For the analysis of pooled recurrent rate, if the study does not provide HR, we manually calculate HR with methods by Tierney et al. [16]. For the analysis of adverse events, we calculate pooled OR, or OR if outcome was available from one study only. The heterogeneity of effect size estimates was assessed using forest plots to detect non-overlapping confidence interval (CI), and then was calculated using the $\mathrm{Q}$ statistic and $\mathrm{I}^{2}$ statistic. For the $\mathrm{Q}$ statistic, substantial heterogeneity was defined as $\mathrm{p}<0.10$. The $\mathrm{I}^{2}$ statistic ranges in value from 0 to $100 \%\left(\mathrm{I}^{2}<25 \%\right.$, low heterogeneity; $\mathrm{I}^{2}=25 \%-50 \%$, moderate heterogeneity; and $\mathrm{I}^{2}>50 \%$, substantial heterogeneity). A sensitivity analysis was performed to assess the influence of the individual studies on the overall results by omitting one study at a time. Publication bias was assessed using funnel plot and Egger's regression tests [19] $(\mathrm{p}<0.05$ was considered significant). All statistical tests were performed using the STATA 14.2 software (College Station, TX).

\section{Results}

\subsection{Search result}

Our search strategy yielded 144 potentially relevant articles (91 articles from PubMed, 53 articles from EMBASE). After the exclusion of 45 duplicate articles, 99 articles underwent title and abstract review. Further, 94 articles were excluded due to at least one of the following reasons: 1) study objective was irrelevant or was not a randomized controlled trial, 2) was not conducted in patients with pAF, 3) did not compare ablation with medical therapy or focus on an ablation technique, or 4) same author group with the same database. This left 5 studies for full manuscript review. All 5 studies met inclusion criteria. No additional articles were added through an additional manual search. Thus, a total of 5 articles were included in the data analysis. The PRISMA flow diagram is shown in figure 1

\subsection{Description of included studies}

Five studies from 2005-2020 involving 985 patients were included (495 patients and 490 patients underwent ablation and medication as initial therapy, respectively) $[10-12,14,15]$. The main inclusion criterion was symptomatic pAF without previous treatment with class I or class III AADs. Exclusion criteria are reported in detail in Table 1. The most commonly used first-line AADs were flecainide, propafenone, and sotalol, with amiodarone a second-line AAD in all studies. AT recurrences were recorded after a blanking period of 60-90 days and up to 1-2 years via ambulatory cardiac monitoring. Other study characteristics of the included studies are shown in table 1.

\subsection{Quality assessment of included studies}

The Cochrane Collaboration tool for assessing risk of bias is shown in table 2 .

\subsection{Meta-analysis results}

Freedom from atrial tachyarrhythmia 
The outcome of AT recurrences was available in all 5 studies [10-12, 14, 15]. Patients who underwent catheter ablation for pulmonary vein isolation had an increased freedom from AT during the 12-24 months follow-up period (pooled $\mathrm{HR}=0.48,95 \%$ CI: $0.40-0.59, \mathrm{p}<0.001, \mathrm{I} 2=0.0 \%$ ) compared to AAD therapy (Figure 2). In the subgroup analysis of ablation method, patients in the cryoablation group (pooled HR $=0.49,95 \%$ CI: 0.38 $0.64, \mathrm{p}<0.001, \mathrm{I} 2=0.0 \%$ ) (Figure $2 \mathrm{~A}$ ) and radiofrequency ablation group (pooled HR=0.47, 95\%CI:0.35-0.64, $\mathrm{p}<0.001, \mathrm{I} 2=0.0 \%$ ) (Figure $2 \mathrm{~B}$ ) both had increased freedom from AT during the 12-24 months follow-up period compared to AAD therapy.

Adverse events

A summary of adverse events from the included studies is shown in table 4. Studies reporting zero event rates in both ablation and AAM group were excluded from the meta-analysis for adverse events. As such, we performed a meta-analysis of adverse events with these available outcomes in at least two of the included studies. There were no differences in adverse events from studies in which data were available for metaanalysis for cerebrovascular accident, PV stenosis, pericardial effusion or tamponade, bradycardia requiring pacemaker, syncope, acute coronary syndrome, deep vein thrombosis (DVT) and pulmonary embolism (PE). Other adverse events that we did not perform meta-analysis included mortality, phrenic nerve injury, esophageal-related complications, and perforation.

\subsection{Publication bias}

We aimed to investigate potential publication bias via the funnel plot and Egger's test. However, as we only had up to 5 studies in the main analysis (figure 2), the number was insufficient to reject the assumption of no funnel plot asymmetry. Thus, we did not perform a funnel plot or Egger's test [20, 21].

\section{Discussion}

The main finding from our updated study that includes contemporary ablation tools is that catheter ablation for pulmonary vein isolation is more effective than $\mathrm{AAD}$ as initial therapy for $\mathrm{pAF}$ in reducing AT recurrences after the initial blanking period to 1-2 years. In addition, this benefit with ablation was the same in both cryoablation and radiofrequency ablation with similar relative reductions in risks. Despite the augmented efficacy for reduction in AF recurrences with catheter ablation, we did not observe a significant increase in any adverse events in these patients compared to AAD therapy.

In this updated meta-analysis of RCTs, we found two recently published RCTs that add to prior summary work in this area. The new search resulted in a total of 5 RCTs to date. The 2 newly added RCTs, EARLY$\mathrm{AF}$ and STOP AF First, used cryoballoon ablation for pulmonary vein isolation, which is different from the previous 3 RCTs that all used radiofrequency ablation. Evidence from the Cryoballoon or Radiofrequency Ablation for Paroxysmal Atrial Fibrillation (FIRE AND ICE) trial suggested that treatment efficacy of cryoballoon ablation was non-inferior to the more traditional radiofrequency ablation in drug-refractory pAF [22]. However, the efficacy of cryoablation in treatment-naïve pAF was still unclear, especially when compared directly to AAD or radiofrequency ablation. The EARLY-AF trial and the STOP AF First trial were the first RCTs to compare cryoablation to AAD as an initial therapy for pAF. This gave us opportunity to indirectly compare the two ablation strategies as initial treatment for pAF. As shown in figure 1, the pooled HR for the cryoablation subgroup (figure 1A) was similar to the pooled HR for the radiofrequency ablation subgroup (figure 2). However, as these pooled data are derived from a retrospective comparison, further prospective RCTs that directly comparing the two techniques are needed to confirm this finding or investigate if one may be preferable over the other for treatment naïve pAF compared to AAD.

The included studies in our meta-analysis demonstrated a significant reduction in AT recurrence at 1-2 years with a similar effect size. The EARLY-AF trial and the RAAFT-2 trial reported HRs for AT recurrence. In the MANTRA-PAF trial, the RAAFT-1 trial, and the STOP-AF First trial, the authors did not report HRs for AT recurrence. Nevertheless, the authors provided sufficient raw data for us to calculate HRs as described in the methods section. The HRs from each study are shown in figure 1 along with the pooled HR.

The study designs are similar among the 5 included RCTs. Inclusion and major exclusion criteria are shown in 
table 1. Briefly, all studies included strictly symptomatic pAF without prior use of class I and III AADs. Major exclusion criteria being used in all studies were previous ablation or surgery of the left atrium, reversible causes of AF, structural or valvular heart disease, a left atrial diameter $>5-5.5 \mathrm{~cm}$, a left ventricular ejection fraction $<35-45 \%$, NYHA class III-IV heart failure. For AT outcome measurements, all studies reported using a blanking period of 60-90 days following the ablation with follow up of patients up to 1-2 years. Three studies used radiofrequency ablation and two studies used cryoablation as described in table 1 . The subgroup analysis by ablation method is shown in figure $2 \mathrm{~A}$ and $2 \mathrm{~B}$ that demonstrates similar results between the 2 ablation techniques. The consistency and similarity in study design likely contributed to the absence of heterogeneity $(\mathrm{I}=0.0 \%)$ in our analysis, which indicates that our result is robust.

Atrial arrhythmia type as endpoint differs slightly among the included studies. The MANTRA-PAF trial and the RAAFT-1 trial considered only AF as the clinical endpoint for recurrence. For the EARLY-AF trial, the RAAFT-2 trial, and the STOP AF First trial, authors consider either of AF, atrial flutter, and atrial tachycardia as the clinical endpoint. It is likely due to the difference in definition of endpoint that the MANTRA-PAF trial and the RAAFT-1 trial reported lower recurrence rates than the other 3 RCTs, except for the AAD group from the RAAFT-1 trial that had a comparable recurrent rate to the other 3 RCTs.

Table 3 shows a summary of the adverse events. Overall, the adverse event rates were low, and not many specific adverse events of interest occurring in either of the ablation group or AAD group in some studies. We were able to perform a meta-analysis on several adverse events including cerebrovascular accident, PV stenosis, pericardial effusion or tamponade, bradycardia requiring pacemaker, syncope, acute coronary syndrome, DVT or PE, all of which were statistically similar between the two groups. For other adverse events including mortality, phrenic nerve injury, esophageal-related complications, and perforation, there was only 1 study available for a meta-analysis for each outcome. The individual ORs for these adverse events also did not demonstrate significant differences between the two groups. Nevertheless, these findings must be interpreted with caution as this could be from inadequate power from the extremely low number of events which subjects this sub-analysis to risk of a type II error.

\section{Limitations}

We acknowledge certain limitations within our study. First, extracted/calculated HRs were not adjusted for confounders. Secondly, there were differences in follow-up times with 3 and 2 studies that followed patients up to 12 and 24 months, respectively. Also, there was a difference in the definition of arrhythmia recurrences used for the clinical endpoint as discussed above. Nevertheless, we did not observe major differences in the HR, and the random-effect model did not reveal significant heterogeneity from our analysis (I $2=0.0 \%$ ). Thirdly, data regarding the adverse event were limited due to the overall low event rates which subsequently limited the power of the analysis.

\section{Conclusions}

In this updated systematic review and meta-analysis of RCTs, that now includes RCTs that specifically use cryoballoon ablation only, we found that catheter ablation is more effective than AADs as an initial therapy for $\mathrm{pAF}$ in reducing AT recurrences over 1-2 years following the treatment initiation. These results are the same with use of both cryoablation and radiofrequency ablation approaches. The adverse event rates are low with contemporary use of AADs and with evolved catheter ablation tools and there were no differences in any adverse events between the two groups.

\section{Acknowledgement}

None

\section{Reference}

1. Andrade J, Khairy P, Dobrev D, Nattel S (2014) The clinical profile and pathophysiology of atrial fibrillation: relationships among clinical features, epidemiology, and mechanisms. Circulation research 114(9):14531468 . 
2. Simantirakis EN, Papakonstantinou PE, Kanoupakis E, et al. (2018) Recurrence rate of atrial fibrillation after the first clinical episode: A prospective evaluation using continuous cardiac rhythm monitoring. Clin Cardiol 41(5):594-600.

3. Dorian P, Paquette M, Newman D, et al. (2002) Quality of life improves with treatment in the Canadian Trial of Atrial Fibrillation. American heart journal 143(6):984-990.

4. Mark DB, Anstrom KJ, Sheng S, et al. (2019) Effect of Catheter Ablation vs Medical Therapy on Quality of Life Among Patients With Atrial Fibrillation: The CABANA Randomized Clinical Trial. Jama 321(13):12751285 .

5. January CT, Wann LS, Alpert JS, et al. (2014) 2014 AHA/ACC/HRS guideline for the management of patients with atrial fibrillation: executive summary: a report of the American College of Cardiology/American Heart Association Task Force on practice guidelines and the Heart Rhythm Society. Circulation 130(23):20712104.

6. Ghia KK, Chugh A, Good E, et al. (2009) A nationwide survey on the prevalence of atrioesophageal fistula after left atrial radiofrequency catheter ablation. Journal of interventional cardiac electrophysiology : an international journal of arrhythmias and pacing 24(1):33-36.

7. Garg L, Garg J, Gupta N, et al. (2016) Gastrointestinal complications associated with catheter ablation for atrial fibrillation. International journal of cardiology 224:424-430.

8. Wilber DJ, Pappone C, Neuzil P, et al. (2010) Comparison of antiarrhythmic drug therapy and radiofrequency catheter ablation in patients with paroxysmal atrial fibrillation: a randomized controlled trial. Jama 303(4):333-340.

9. Packer DL, Kowal RC, Wheelan KR, et al. (2013) Cryoballoon ablation of pulmonary veins for paroxysmal atrial fibrillation: first results of the North American Arctic Front (STOP AF) pivotal trial. J Am Coll Cardiol 61(16):1713-1723.

10. Morillo CA, Verma A, Connolly SJ, et al. (2014) Radiofrequency ablation vs antiarrhythmic drugs as first-line treatment of paroxysmal atrial fibrillation (RAAFT-2): a randomized trial. JAMA 311(7):692-700.

11. Cosedis Nielsen J, Johannessen A, Raatikainen P, et al. (2012) Radiofrequency ablation as initial therapy in paroxysmal atrial fibrillation. N Engl J Med 367(17):1587-1595.

12. Wazni OM, Marrouche NF, Martin DO, et al. (2005) Radiofrequency ablation vs antiarrhythmic drugs as first-line treatment of symptomatic atrial fibrillation: a randomized trial. JAMA 293(21):2634-2640.

13. Hakalahti A, Biancari F, Nielsen JC, Raatikainen MJ (2015) Radiofrequency ablation vs. antiarrhythmic drug therapy as first line treatment of symptomatic atrial fibrillation: systematic review and meta-analysis. Europace 17(3):370-378.

14. Andrade JG, Wells GA, Deyell MW, et al. (2020) Cryoablation or Drug Therapy for Initial Treatment of Atrial Fibrillation. N Engl J Med;10.1056/NEJMoa2029980.

15. Wazni OM, Dandamudi G, Sood N, et al. (2020) Cryoballoon Ablation as Initial Therapy for Atrial Fibrillation. N Engl J Med;10.1056/NEJMoa2029554.

16. Tierney JF, Stewart LA, Ghersi D, Burdett S, Sydes MR (2007) Practical methods for incorporating summary time-to-event data into meta-analysis. Trials 8:16.

17. Higgins JP, Altman DG, Gotzsche PC, et al. (2011) The Cochrane Collaboration's tool for assessing risk of bias in randomised trials. BMJ 343:d5928.

18. DerSimonian R, Laird N (1986) Meta-analysis in clinical trials. Control Clin Trials 7(3):177-188.

19. Sterne JA, Egger M (2001) Funnel plots for detecting bias in meta-analysis: guidelines on choice of axis. J Clin Epidemiol 54(10):1046-1055. 
20. Simmonds M (2015) Quantifying the risk of error when interpreting funnel plots. Systematic reviews $4: 24-24$.

21. Debray TPA, Moons KGM, Riley RD (2018) Detecting small-study effects and funnel plot asymmetry in meta-analysis of survival data: A comparison of new and existing tests. Research synthesis methods $9(1): 41-50$.

22. Kuck KH, Brugada J, Furnkranz A, et al. (2016) Cryoballoon or Radiofrequency Ablation for Paroxysmal Atrial Fibrillation. N Engl J Med 374(23):2235-2245.

Table 1: Study characteristics of included randomized controlled trials

Table 2: Basic characteristics of study participants

Table 3: Cochrane Collaboration tool to assess risk of bias for randomized controlled trials

Table 4: Summary of adverse events

Fig. 1: PRISMA flow diagram.

Fig. 2: Forest plot of the included studies comparing arrhythmia-free survival between ablation group and antiarrhythmic medication group; 2A: Cryoablation, 2B: Radiofrequency ablation

Supplementary material 1: Search term

\section{Hosted file}

Table 1.pdf available at https://authorea.com/users/315715/articles/515988-ablationvs-medication-as-initial-therapy-for-paroxysmal-atrial-fibrillation-an-updated-metaanalysis-of-randomized-controlled-trials

\section{Hosted file}

table 2.pdf available at https://authorea.com/users/315715/articles/515988-ablationvs-medication-as-initial-therapy-for-paroxysmal-atrial-fibrillation-an-updated-metaanalysis-of-randomized-controlled-trials

\section{Hosted file}

table 3. cochrane.pdf available at https://authorea.com/users/315715/articles/515988-ablationvs-medication-as-initial-therapy-for-paroxysmal-atrial-fibrillation-an-updated-metaanalysis-of-randomized-controlled-trials

\section{Hosted file}

table 4 complication.pdf available at https://authorea.com/users/315715/articles/515988ablation-vs-medication-as-initial-therapy-for-paroxysmal-atrial-fibrillation-an-updatedmeta-analysis-of-randomized-controlled-trials 

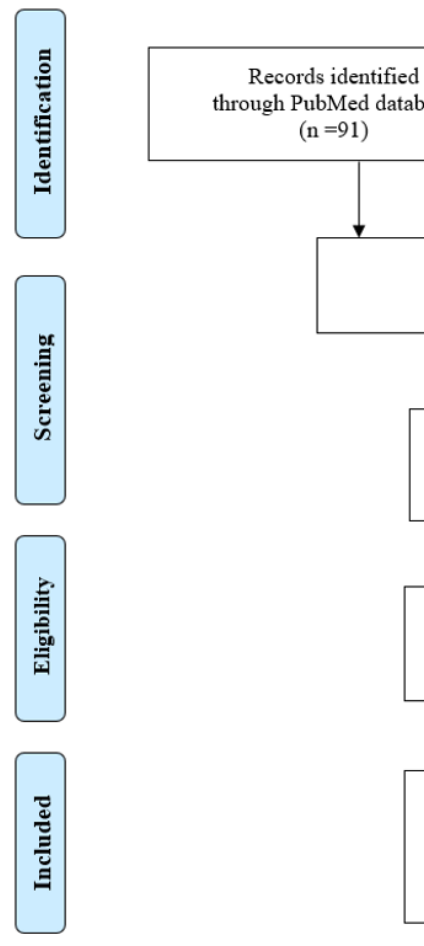
through PubMed database

$$
(\mathrm{n}=91)
$$

Additional records identified through EMBASE database $\mathrm{n}=91$ ) $(\mathrm{n}=53$

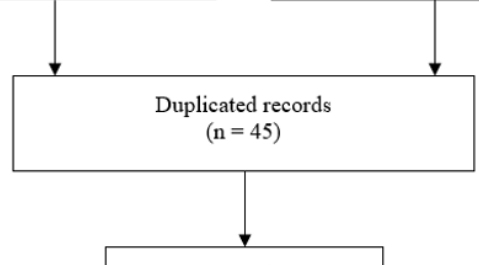

Records excluded (94) due to - irrelevant objective or was not a clinical trial (77) are with medical therapy or focus

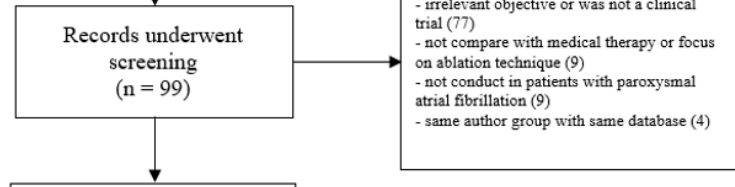

Full-text articles assessed for eligibility

$$
(\mathrm{n}=5)
$$

No full-text articles were excluded

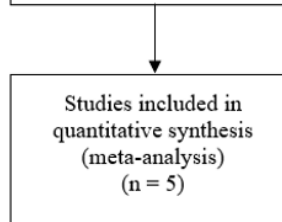

First author, year

HR (95\% Cl)

Weight (\%)

A Andrade et al., 2020

Wazni et al., 2020

Subtotal (I-squared $=0.0 \%, p=0.749$ ) $z=5.29, p<0.001$ B

Morillo et al., 2014 Nielsen et al., 2012 Wazni et al., 2005

Subtotal (I-squared $=0.0 \%, p=0.418$ ) $z=4.85, p<0.001$

Overall (I-squared $=0.0 \%, p=0.757$ ) $\mathrm{z}=7.17, \mathrm{p}<0.001$ NOTE: Weights are from random effects analýsis

$\longleftarrow$ Favors ablation 1 Favors antiarrhythmic $\longrightarrow^{10}$

$0.48(0.35,0.65) \quad 42.92$

$0.53(0.31,0.90) \quad 14.18$ $0.49(0.38,0.64) \quad 57.10$

$0.56(0.35,0.90) \quad 17.68$ $0.48(0.30,0.78) \quad 16.82$ $0.32(0.16,0.63) \quad 8.40$ $0.47(0.35,0.64) \quad 42.90$ $0.48(0.40,0.59) \quad 100.00$ 